\title{
DualBeam Slice \& View: practical aspects for collecting 3D cortex image data
}

\author{
J.J.L. Mulders*, G. Knott**, B.H. Lich* \\ * FEI Electron Optics, Achtseweg Noord 5, 5600 KA Eindhoven, The Netherlands \\ ** DBCM, Faculté de Medicine, Université de Lausanne, Switzerland CH1005
}

To enable the study of the $3 \mathrm{D}$ architecture of cortex tissue it is necessary to create an image data set with sufficient detail in all directions. One way to provide a data set is to apply mechanical slicing and TEM imaging, however, this process is tedious and requires a lot of manual input and control therefore it is time consuming and very labor intensive. Another, novel way to create a data set of the cortex, is to apply a FIB based slicing with successive in-situ SEM based high-resolution imaging. Although slice and view applications on such a DualBeam system have generally been automated since a few years, practical limits in terms of timing, signal / noise levels and image resolution need to be tuned specifically for the purpose of cortex imaging. In general the total process for 3D imaging of the cortex is easy to describe:

1. Prepare the sample and create a FIB aligned edge at the volume of interest.

2. Define milling conditions and patterning definitions for the proper volume. Match the slice thickness (resolution in z-direction) with lateral x,y image resolution.

3. Select an imaging detector, to be applied after the slicing process and define the imaging conditions in terms of pixel range and dwell times..

4. Run the successive slicing and viewing process in an automated way.

For the process indicated above, but applied to the parameter setting for the cortex data set, some conditions and limitations have to be studied in more detail.

1) The volume of interest

The volume to be analyzed is given by the characteristic length of the features of interest. For this reason, although many small features such as vesicles of $30-50 \mathrm{~nm}$ have to be visible, the actual dimension of the volume is in the range of $100 \times 100 \times 100 \mathrm{um}$. For any high resolution FIB column, this is a large volume, as the slice milling yield for this kind of sample typically is around 1.8 $\mathrm{um}^{3} / \mathrm{nC}$, whereas the required slice thickness (resolution of the third dimension) allows for a maximum beam current of $1 \mathrm{nA}$. This would imply a total milling time of $10^{+6} / 1.8 \times 1=154 \mathrm{hrs}$.

2) Detector choice and position

As the sample has been fixed and stained, the application of back-scatter contrast is best suited to allow for an adequate signal to noise ratio. Note that, due to the nature of the milling process, the cross-sectional surface is actually very smooth, so the contribution of any topography is low. Imaging with a secondary electron detection system basically shows the contribution of SE type II, rather then type I. For this reason it is better to allow direct imaging with the back-scatter electrons, applying an adequate solid-state BS detector, in addition to a relative low energy (3-5 keV) for the electron beam. Note that, due to the viewing angle, a through-the-lens BS detector is less suited. Because the sample is continuously in the milling position, the detection of back-scattered electrons has to be optimized for collection of signal in this (fixed) sample position. For the optimization of the process this total collection angle is quite critical for the signal strength, as shown in figure 1, for a metal test sample in the milling position and the BS detector at various positions with fixed, equal distances. 


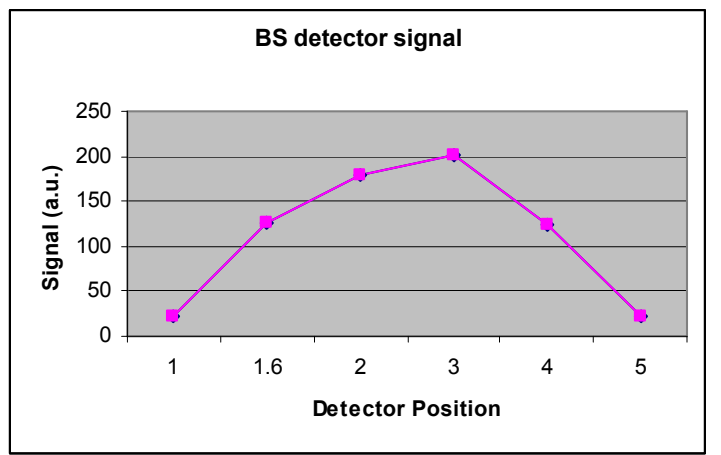

Figure 1: The relative SS BS signal strength at various positions opposite the cross-section as measured on a metal test sample is shown in the figure on the left.

From this graph the sensitivity of the BS detector position to the signal strength is very clear. The resulting image quality, shown below, was obtained with the detector yet at the non-ideal position 1.6.

\section{3) Milling strategy}

It should be noted that the BS collection angle is a two dimensional parameter and that signal opening angle towards the detector (yield) must be equal for all slices. The last slice however - deep in the material - has a smaller detection yield, because the edges produce a shadowing area for the signal in the direction of the detector. With a proper milling strategy this problem can be eliminated. A second issue to consider is that the linear dimension of the volume aimed for is larger then the beam shift range for the ion column, and so it will be impossible to apply a continuous, subsequent milling process. At some point, the milling process has to be interrupted to allow for stage shift and simultaneous reset of the ion beam shift. Of course, with extremely high-accuracy re-positioning.

\section{4) Imaging strategy}

To speed up the process as much as possible, the image collection speed is critical. Assuming a $4 \mathrm{kx} 4 \mathrm{k}$ image at 10 us / pixel the collection time of 160 seconds per image will result in a total imaging time of 111 hours. An example of the image quality (signal to noise) at this image collection speed but at higher magnification is given in the figure 2 .

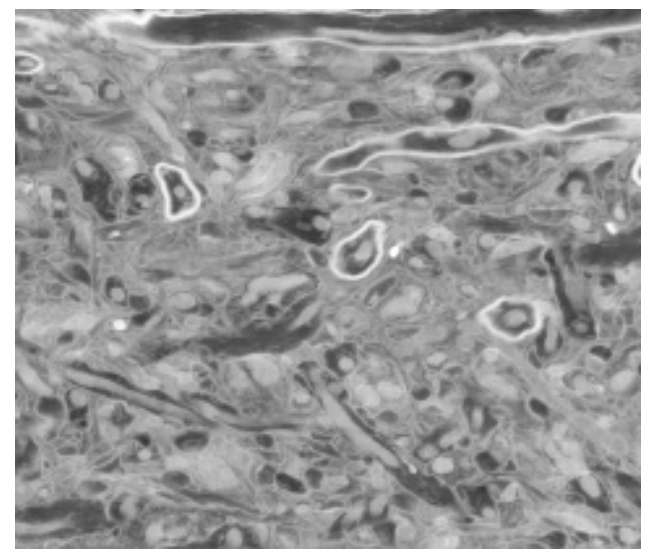

Figure 2: Image of mouse brain cortex at mill location.. $\mathrm{HFW}=13 \mathrm{um}, 5 \mathrm{keV}$ BS image, detector position 1.6.

This image quality is considered adequate. However, a 4 x 4 $\mathrm{k}$ image at 100 um field width would provide a pixel size of $24 \mathrm{~nm}$, which is too large. Therefore it is suggested to use at least four images to cover the full area. In addition, by optimizing the detector position according to Figure 1 (above) the pixel dwell time can be further reduced to 3 us. This will lead to a total image collection time of 100/0.04 (slices) $\times 192($ sec/slice $)=133 \mathrm{hrs}$.

\section{5) Conclusion}

Within the boundaries mentioned above, for this application the potential run time can be in the range of 300 hours: much faster then the TEM approach. It will require a step wise approach to allow for image stitching per slice as well as ion beam shift reset followed by stage move compensation. As a large part of the process can be automated the majority of the data can be collected without operator interventions. The total process has a step-wise approach with limited operator intervention, so the instrument is not completely blocked for a long time for this application only. 